\title{
POLAR: Instrument and Results
}

\author{
Brian G. Keating*, Angelica de Oliveira-Costa ${ }^{\dagger}$, Christopher W. O’Dell ${ }^{* *}$, Lucio \\ Piccirillo $^{\ddagger}$, Nate C. Stebor ${ }^{\S}$, Max Tegmark ${ }^{\dagger}$ and Peter T. Timbie ${ }^{* *}$ \\ ${ }^{*}$ Division of Physics, Math, and Astronomy, California Institute of Technology, Pasadena, CA 91125 \\ ${ }^{\dagger}$ Department of Physics, University of Pennsylvania, Philadelphia, PA 19104 \\ ${ }^{* *}$ Department of Physics, University of Wisconsin - Madison, Madison, WI 53706 \\ ${ }^{\ddagger}$ Department of Physics and Astronomy, University of Wales - Cardiff, Wales, UK CF24 $3 Y B$ \\ ${ }^{\S}$ Department of Physics, University of California at Santa Barbara, Santa Barbara, CA 93106
}

\begin{abstract}
We describe the design, performance, and results of a polarimeter used to make precision measurements of the $2.7 \mathrm{~K}$ cosmic microwave background. In the Spring of 2000 the instrument searched for polarized emission in three microwave frequency bands spanning 26-36 GHz. The instrument achieved high sensitivity and long-term stability, and has produced the most stringent limits to date on the amplitude of the large angular scale polarization of the cosmic microwave background radiation.
\end{abstract}

URL http://cmb.physics.wisc.edu/polar

\section{INTRODUCTION}

The 2.7K Cosmic Microwave Background (CMB) radiation is a vital probe of all modern cosmological theories. This radiation provides a "snapshot" of the epoch at which radiation and matter decoupled, approximately 300,000 years after the Big Bang, and carries the imprint of the ionization history of the universe. This information has been used to tightly constrain theories of cosmological structure formation, and has ushered in the era when "cosmological accuracy" is no longer a pejorative term.

Three properties are necessary to fully characterize the CMB: its spectrum, spatial isotropy, and polarization. The first two properties have been measured, whereas the polarization state of the CMB remains undetected. Detection of, or an improved upper limit on, the polarization of the CMB at large scales holds great promise for the determination of several fundamental properties of the standard cosmological model, such as the ionization history of the Universe and the contribution of gravitational waves to the spectrum of primordial perturbations. Most models predict that the magnitude of the polarization of the $\mathrm{CMB}$ at large angular scales is less than $1 \mu \mathrm{K}$. This is at least an order of magnitude below both the large scale anisotropy level of the CMB, as well as the best existing upper limits on its polarization.

Similar to the CMB anisotropy power spectrum, the polarization power spectrum contains information on all angular scales. Large angular scales (larger than $\simeq 1^{\circ}$ ) correspond to regions on the last scattering surface which were larger than the causal horizon at $z \simeq 1000$. In the absence of reionization, these scales were affected only by the long wavelength modes of the primordial power spectrum. This region of the anisotropy power spectrum was measured by the COBE DMR, and establishes the normalization for models of large scale structure formation. Similarly, measurements of polarization at large angular scales will normalize the entire polarization power spectrum. Because the anticipated signal size is small at all angular scales, polarization measurements face more formidable challenges than anisotropy measurements.

The experiment described here measured polarization on large angular scales. While these signals may be weaker than signals on small scales, the design of a large angular scale measurement is comparatively simple and compact, with potentially lower susceptibility to sources of systematic error. This report describes the design of, and results from, Polarization Observations of Large Angular Regions (POLAR). 
TABLE 1. POLAR $K_{\mathrm{a}}$-Band Measured and Modeled FWHM Beam Widths.

\begin{tabular}{|c|c|c|}
\hline Plane & $v[\mathrm{GHz}]$ & $\theta_{f w h m} \pm 0.1^{\circ}$ \\
\hline $\mathrm{E}$ & 26 & $7.5^{\circ}$ \\
\hline $\mathrm{E}$ & 29 & $7.0^{\circ}$ \\
\hline E & 36 & $6.4^{\circ}$ \\
\hline $\mathrm{H}$ & 29 & $7.1^{\circ}$ \\
\hline $\mathrm{H}$ & 36 & $6.5^{\circ}$ \\
\hline
\end{tabular}

\section{INSTRUMENT}

The POLAR radiometer is comprised of three main sections:

- Cold receiver components: optics, OMT, isolators, HEMT amplif iers.

- Room-temperature receiver components: warm RF amplif iers, heterodyne stage, warm IF amplif iers, banddef ining filters, correlators.

- Post-detection components: pre-amplif iers, low frequency processing, and data acquisition.

POLAR is a superheterodyne correlation polarimeter that measures two orthogonal linear polarization states in three radiofrequency (RF) bands in the $K_{\mathrm{a}}$-band between $26-36 \mathrm{GHz}$. In many respects, the signal processing techniques used in correlation polarimeters are similar to those used in polarization sensitive interferometers. The two polarization states $i \in\{x, y\}, E_{i}^{R F}\left(t, v, \phi_{i}\right)=E_{i} \cos \left(2 \pi v t+\phi_{i}\right)$ enter a single-mode circular corrugated feedhorn and are separated by an orthomode transducer (OMT). The OMT's polarization isolation $(-35 \mathrm{~dB})$ and low cross-polarization $(-30$ $\mathrm{dB}$ ) ensure low spurious polarization. The two polarizations are amplif ied by separate HEMT amplif iers [1] cooled to $25 \mathrm{~K}$ by a mechanical cryocooler. Downconversion from the RF band to an intermediate frequency (IF) band (2$12 \mathrm{GHz}$ ) is performed by Schottky diode mixers, driven by a Gunn diode local oscillator (LO) at $38 \mathrm{GHz}$. In the IF band the two polarization states are amplif ied producing $E_{i}^{I F} \propto E_{i}^{R F}$, and filtered into three separate IF bands, denoted J1, J2, and J3. The IF bands translate into RF bands: J1 (32-36 GHz), J2 (29-32 GHz), and J3 (26-29 GHz). Prior to filtering, two dedicated diode detectors measure the total power of each polarization state, which serve as atmospheric opacity monitors. The fields $E_{x}^{I F}$ and $E_{y}^{I F}$ are correlated by three Schottky Diode analog multiplier (correlator) circuits. The phase of the LO is switched between 0 and $\pi$ at $1 \mathrm{KHz}$ prior to mixing the $E_{y}^{R F}$ waveform. The voltage produced by the correlators at this stage switches between $\kappa E_{x}^{R F} E_{y}^{R F}$ and $-\kappa E_{x}^{R F} E_{y}^{R F}$ at $1 \mathrm{KHz}$, where $\kappa$ converts intensity to voltage. Phase-sensitive detection of this modulated signal reduces the effects of low frequency drifts in the LO output power and/or correlator sensitivity to negligible levels. After low-pass filtering, we record an audio-band signal with a DC component $I_{D C}=\kappa\left\langle E_{x}^{R F} E_{y}^{R F}\right\rangle$, where the brackets denote a time-average, and AC components proportional to the thermal noise from the radiometer, atmosphere, and celestial signals. These signals are referred to as the "science channels". A second lock-in amplif ier for each correlator is referenced to the same 1 $\mathrm{KHz}$ waveform but delayed in phase by $\pi / 2$ with respect to the phase switch. These signals, hereafter referred to as "quadrature phase channels" (QPC), contain only the noise terms of the RF band and no optical or celestial signals. The QPC are powerful probes of systematic effects produced solely by the radiometer and post-detection stages. The output from the QPC is proportional to the noise equivalent temperature (NET) of the instrument. For a correlation radiometer this is NET $=\sqrt{2 / \Delta v}\left(\mathrm{~T}_{\mathrm{Rx}}+\mathrm{T}_{\mathrm{Ant}}\right)\left[\mathrm{K}-\mathrm{s}^{1 / 2}\right]$, where $\Delta v$ is the bandwidth of the radiometer, $T_{\mathrm{Rx}}$ is the receiver noise temperature, and $T_{\mathrm{Ant}}$ is the antenna temperature of observed optical sources, including diffuse sources such as the atmosphere and the CMB itself. A schematic outline of POLAR is presented in Figure 1.

POLAR uses two levels of ground screening. First an inner conical ground screen rotates with the instrument and is coated with Eccosorb. The Eccosorb panels absorb, rather than redect the sidelobes to the sky. We estimate the antenna temperature of the inner shield to be $<1 \mathrm{~K}$. Polarization generated by emission from the bare metal surface of the uncovered shield is believed to be much more troublesome than the slight increase in system temperature. POLAR's inner ground screen co-rotates with the receiver, which ensures that if there is any residual polarized power produced by the inner screen, it would produce a constant polarized offset, which is subtracted during the analysis. The second level of shielding is of the more conventional redective-scoop design, e.g. Wollack [2]. The scoop is mounted to the 


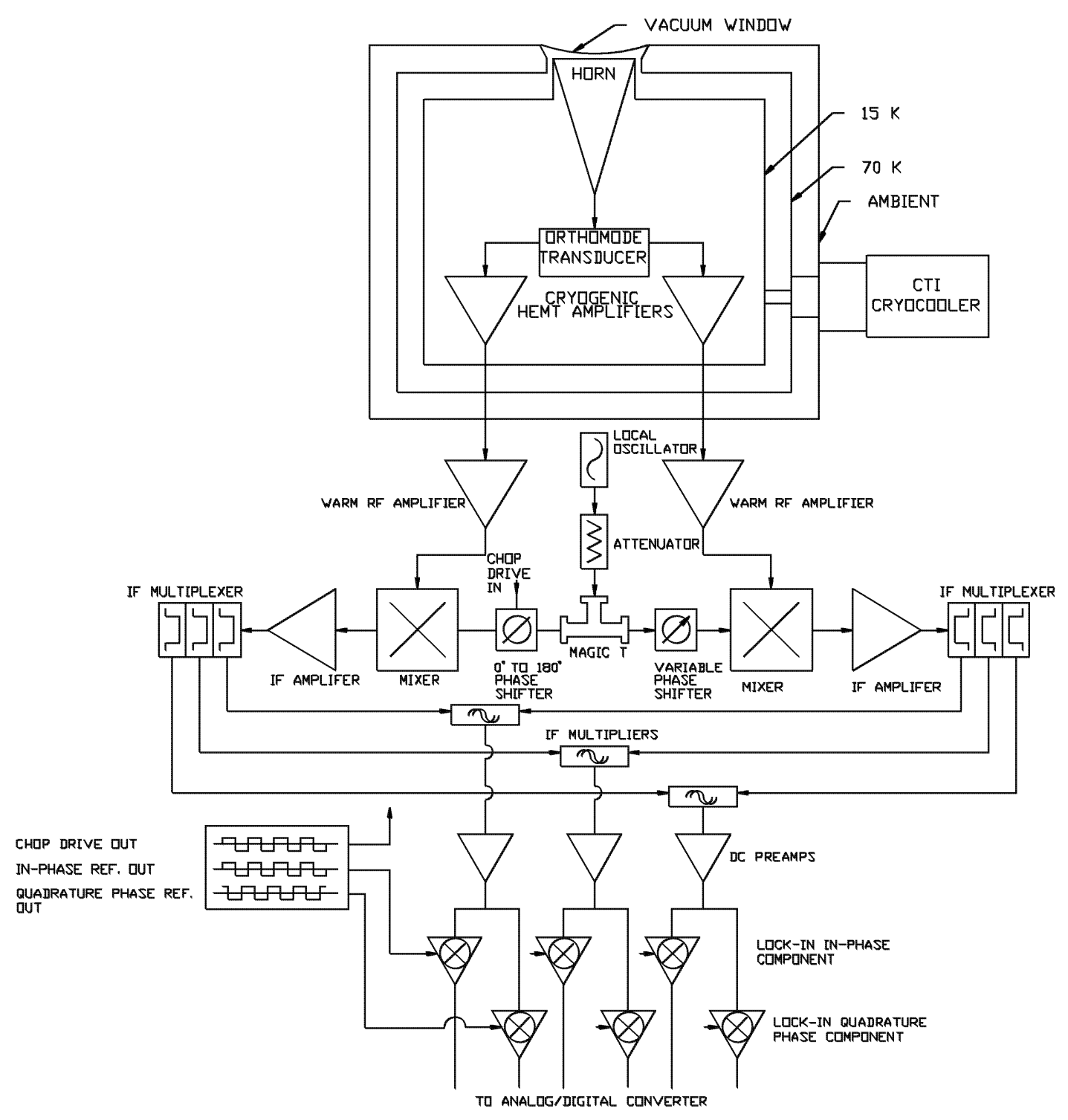

FIGURE 1. POLAR radiometer schematic.

side of the POLAR observatory, and is made of aluminum panels, 8' wide and 6' high. We estimate the level of sidelobe suppression to be $\sim-40 \mathrm{~dB}$.

\section{SITE}

POLAR's observations are conducted from a custom-built observatory located at the University of Wisconsin's Pine Bluff Observatory, (PBO). PBO is located at Longitude $+89^{\circ} 45^{\prime}$, Latitude $+43^{\circ} 01^{\prime}$, approximately 10 miles west of the campus and downtown Madison. It was determined that $\mathrm{PBO}$ was a relatively RF quiet region.

A motorized dome encloses the radiometer and rotating ground screen, keeping precipitation out, and maintaining a moderately thermally stabilized enclosure. The dome itself can be operated manually, or remotely via a WWW page in 


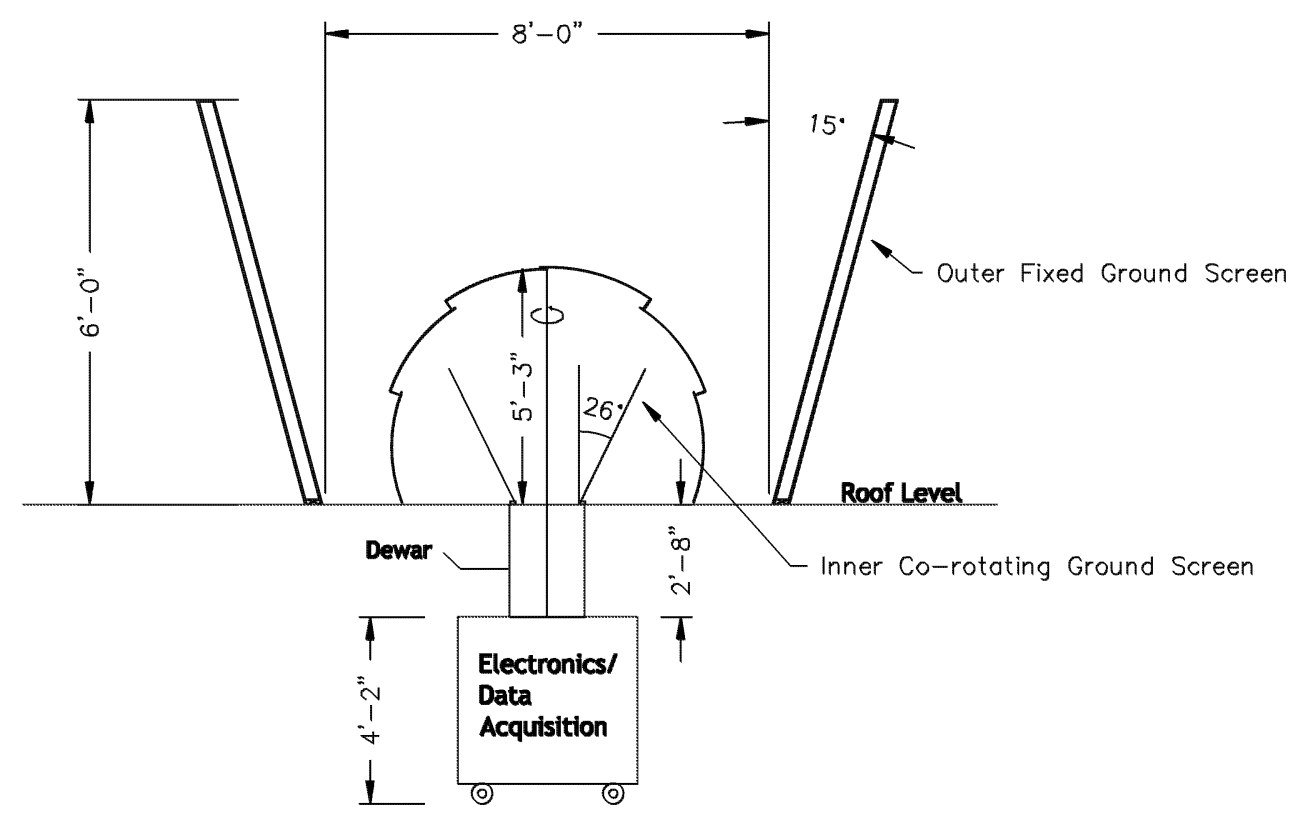

FIGURE 2. POLAR observatory. Two sets of ground screens are used to reduce the polarized spillover from the earth, as well as polarized emission from the shields themselves. The outer shield is fixed to the structure in which PoLAR resides, and is composed of a lightweight steel skeleton covered by 0.05 " aluminum sheets. The inner ground screen is covered with Dat Eccosorb panels, and co-rotates with the radiometer. Also shown is the motor-driven, fiberglass clamshell-dome which is remotely operated via the World Wide Web in the event of inclement weather. The rotation mount, drive motor, bearing, and angular encoder are also shown.

TABLE 2. Measured Properties of POLAR's OMT: Atlantic Microwave Model 2800.

\begin{tabular}{|c|c|c|}
\hline | & Property & Value \\
\hline | & Isolation & $-35 \mathrm{~dB}$ \\
\hline & VSWR & $<1.2$ \\
\hline & s-polarization & $-30 \mathrm{~dB}$ \\
\hline
\end{tabular}

case of inclement weather developing while the experimentalist is elsewhere. The platform has a high-voltage power supply for operation of the CTI 8500 compressor, which requires $220 \mathrm{~V}$ at roughly $10 \mathrm{~A}$. Also running to and from the pad is an Ethernet hub and cables which provide an intranet for data to be transferred from the rotating computer attached to the radiometer to a desktop workstation located $\sim 100^{\prime}$ away in a separate building.

\section{SYSTEMATIC EFFECTS}

Because the anticipated polarization signal is a factor of $\leq 10$ times smaller than the temperature anisotropy currently being detected, a thorough understanding of systematic errors is crucial. Polarimeters, such as POLAR have several advantages, however, that promise to make this effort possible. First, the atmosphere is known to be polarized only at a very low level; far below the expected level of CMB polarization [3]. Additionally, PoLAR mapped polarization in a manner which de-correlated signals from neighboring pixels. POLAR compared the orthogonal polarization signals through the same airmass, and at the same time. In anisotropy observations, beam switching often adds noise and additional chop-dependent signals. Atmospheric effects had a smaller contribution to POLAR than to 
TABLE 3. POLAR $K_{\mathrm{a}}$ Band Radiometer Components

\begin{tabular}{|c|c|c|}
\hline Device & Manufacturer & Model \\
\hline Circular-Square Transition & Custom Microwave & -- \\
\hline OMT & Atlantic Microwave & OM2800 \\
\hline HEMTs & NRAO & $\mathrm{A} 29$ \& A30 \\
\hline Warm RF Amps & MITEQ & JS426004000-30-8P \\
\hline Mixers & MITEQ & TB0440LW1 \\
\hline Gunn Oscillator & Millimeter Wave Oscillator Co. & -- \\
\hline Warm IF Amps & MITEQ & AFS6-00101200-40-10P-6 \\
\hline Triplexers & Reactel & $-\cdots$ \\
\hline Correlators & MITEQ & DBP112HA \\
\hline Total Power Detectors & Hewlett Packard & HP 8474C \\
\hline Lock-In Amplif iers & Analog Devices & AD630 \\
\hline$K_{\mathrm{a}}$ band Phase Switch & Pacif ic Millimeter Products & $-\cdots$ \\
\hline Dewar & Precision Cryogenic Systems & $-\cdots$ \\
\hline Cryocooler & CTI Cryogenics & 8500 Compressor, 350 Cold Head \\
\hline
\end{tabular}

ground-based CMB anisotropy experiments, and allowed for longer observation times. Long-term observations were key to understanding and removing systematic effects [4]. Many spurious instrumental effects were isolated from astrophysical effects by long-term integration tests with the horn antenna replaced by a cold termination.

The most troubling aspect of these effects is that they may not be stable in time. For the correlator channels, the most pernicious contribution arises from the conversion eff iciency and phase stability of the heterodyne stage. The conversion eff iciency (loss) of the mixers is dependent on the Gunn Oscilator power. The oscillator's output power auctuates (similar to an amplif ier's gain), and therefore introduces mixer gain Ouctuations, which can be misinterpreted as signals.

The relative phasing between the two arms of the radiometer is equally troublesome [5] since phase mismatch between the two RF paths reduces the effective bandwidth. There are several standard methods to improve the stability of the heterodyne stage of the receiver, including phase modulation at frequencies of $\sim 1 \mathrm{KHz}$, and phase-locked loops to stabilize the LO. The latter is quite common in interferometers, though it is not incorporated in the POLAR $K_{\mathrm{a}}$-band system. The former technique was performed for POLAR. In Table 4 we list some important systematic effects encountered in previous polarization measurements and summarize the solution adopted by POLAR.

TABLE 4. Expected Systematic Effects

\begin{tabular}{|lcr|}
\hline Effect & Origin & Control Method \\
\hline Mechanical Strain & Instrument Rotation & Zenith Drift Scan \\
\hline Magnetic Coupling & Rotation in $\overrightarrow{\mathbf{B}}_{\text {earth }}$ & Minimal Ferrite Components (Isolators Only) \\
\hline Microphonics & Mechanical Vibration & Vibration Isolation \\
\hline EMI and RFI & Local Sources & Shield/Filter \\
\hline Thermal Variations & Diurnal/Environment & Temp Control \\
\hline Sidelobe Pickup & Sun/Moon/Earth & Low Sidelobe Antenna and Ground Screens \\
\hline
\end{tabular}

\section{OBSERVATION STRATEGY AND SKY COVERAGE}

Over a single night, POLAR swept out a $7^{\circ} \times 360^{\circ} \times \cos 43^{\circ}=1844^{\circ} \mathrm{FWHM}$ swath of the sky. Thirty-six $7^{\circ} \mathrm{FWHM}$ pixels comprise $5 \%$ of the sky. The data is binned into Stokes $Q$ and U vs. RA, and multiple nights of data are co-added. The scan passes through the galaxy twice per day at RA $~ 19 h$ and again at RA $\sim 6 h$. 

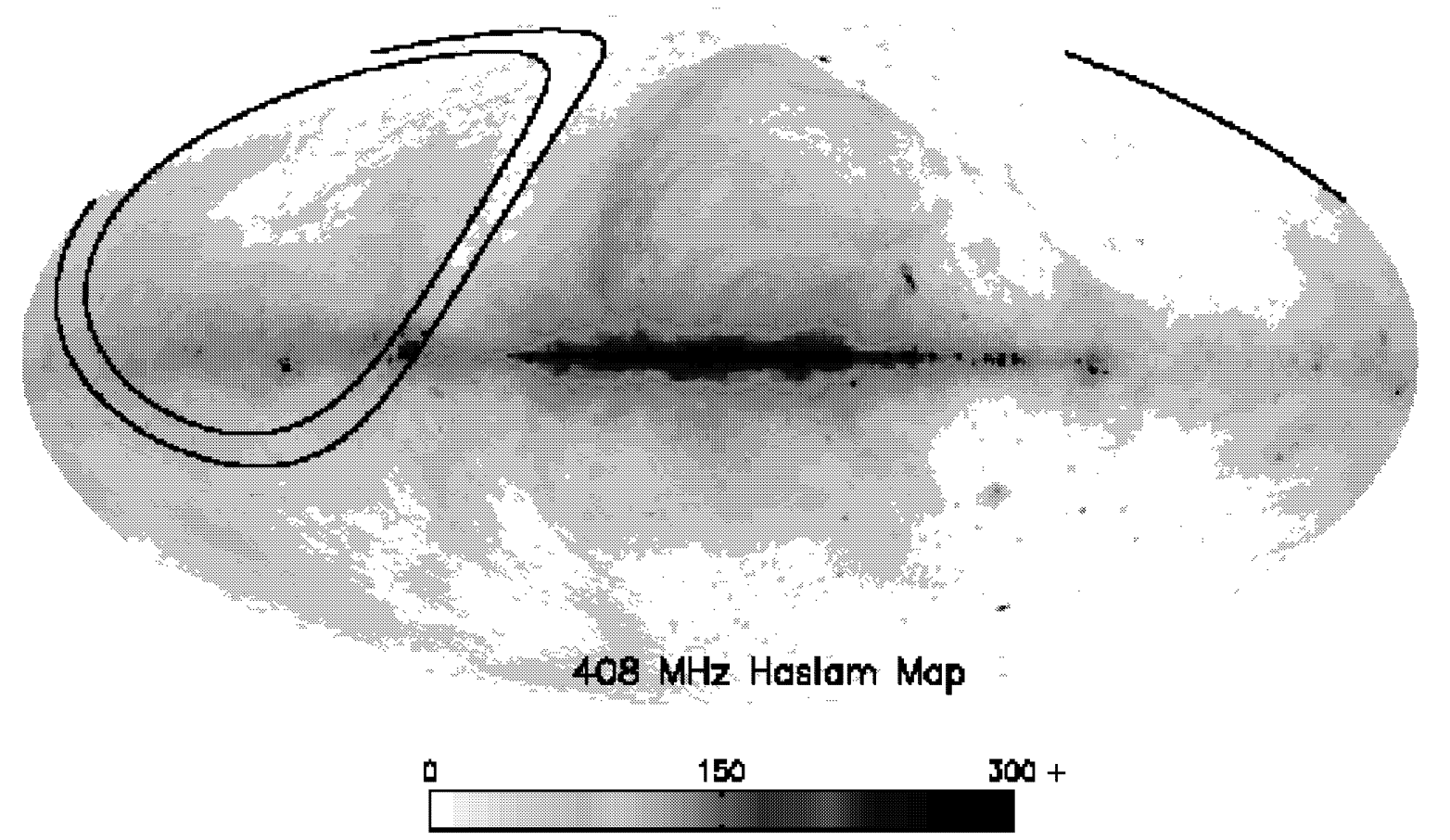

\section{Signal [Kr|tin]}

FIGURE 3. POLAR scan strategy in galactic coordinates, superimposed on the Haslam $408 \mathrm{MHz}$ map

TABLE 5. POLAR Observing Parameters

\begin{tabular}{|c|c|}
\hline Parameter & POLAR 2000 \\
\hline Declination of Drift Scan & $43.03^{\circ}$ \\
\hline Beam width & $7^{\circ} \mathrm{FWHM}$ \\
\hline Fractional Sky coverage & $255^{\circ} \times 7^{\circ} \mathrm{FWHM} \simeq 5 \%$ \\
\hline Rotation Rate & $0.033 \mathrm{~Hz}$ \\
\hline Point Source Sensitivity & $2.8 \mu K \mathrm{Jy}^{-1}$ \\
\hline Post detection Bandwidth & $5 \mathrm{~Hz}$ \\
\hline
\end{tabular}

\section{ATMOSPHERIC EFFECTS}

Although the atmosphere is not expected to produce appreciable linearly polarized radiation, it produces a nonnegligible contribution to the system temperature of the radiometer. Additionally, signif icant fluctuations of atmospheric loading increase the low-frequency noise spectrum of the receiver. We summarize the contribution to the antenna temperature seen by the radiometer in the $K_{\mathrm{a}}$-band by computing the power spectrum of the atmosphere using a commercial code, $\mathrm{AT}^{1}$. To compute the antenna temperature AT requires as input the desired level of precipitable water vapor (PWV). With this specif ied, AT can compute the antenna temperature vs. frequency using a standard model of the earth's atmosphere. Figure 4 shows the atmospheric antenna temperature vs. frequency for various levels of PWV. Since the dominant contribution to the atmospheric temperature comes from the $22 \mathrm{GHz}_{2} \mathrm{O}$ line rather than on the $\mathrm{O}_{2}$ line at $\sim 60 \mathrm{GHz}$, the dependence on PWV is quite noticeable.

\footnotetext{
1 Airhead Software: Boulder, CO
} 


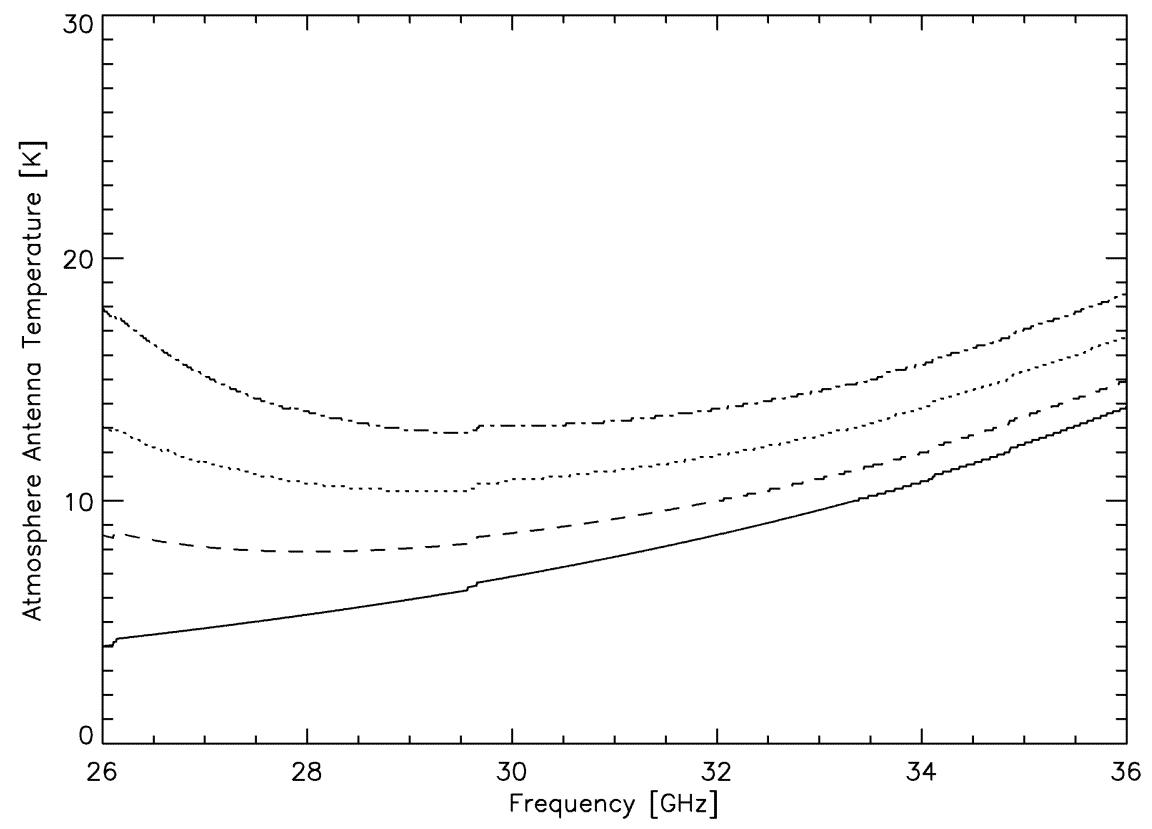

FIGURE 4. Calculated spectrum of Atmospheric Antenna Temperature in the $K_{\mathrm{a}}$-band vs. Precipitable Water Vapor (PWV) . From the top down, the four levels of PWV are: $30 \mathrm{~mm}, 20 \mathrm{~mm}, 10 \mathrm{~mm}, 0 \mathrm{~mm}$. For $50 \%$ of the 2000 observing season, the atmospheric antenna temperature was $<16 \mathrm{~K}$.

\section{OBSERVATIONS AND ANALYSIS}

The PolAR campaign collected a total of 746 hours of data between 2000 March 11 and 2000 May 29. We calibrated the polarimeter by rotating a 0.003 inch dielectric sheet (tilted at $45^{\circ}$ with respect to the optical axis) around the optical axis [6]. This produced a $\sim 500 \mathrm{mK}$ polarized signal. In our coordinate system, def ined with respect to the local geographic coordinates, we performed a $\chi^{2}$ minimization of the angular binned data $f$ it to the function

$$
I\left(\theta_{t}\right)=I_{o}+C \cos \theta_{t}+S \sin \theta_{t}+Q \cos 2 \theta_{t}+U \sin 2 \theta_{t},
$$

where $\theta_{t}=2 \pi f t$. In addition to the Stokes parameters $Q$ and $U$, the terms $C$ and $S$ (which are synchronous with the rotation at frequency $f$ ) were monitored to determine our sensitivity to rotation-synchronous systematic effects, and to monitor atmospheric Ductuations. Three levels of diagnostics were used to detect and remove contaminated data:

- housekeeping and weather (dewpoint, cloud cover) cuts

- time ordered data (TOD) cuts

- rotation ordered data (ROD) cuts after constructing the Stokes parameters.

\section{RESULTS}

For each data segment that passed the data quality tests, the time-ordered data were converted to maps and covariance matrices via the minimum variance map-making procedure outlined in Tegmark [7]. The 25 total sections ranged in length from 4-24 hours. An overall offset was removed from each section, and the data covariance matrix was adjusted accordingly [7, 8]. All sections were combined into a f inal map and covariance matrix for each channel. Keating $e t$ al.(2001) [9] presents the maps of the Stokes parameters for all correlator channels versus RA.

To test the hypothesis that the maps were contaminated by foreground emission, we compared the likelihood for the null-hypothesis of no signal on and off the galactic plane. We tested a simple model of foreground contamination by a single Quctuating component [10]. Tests on these sections of the maps indicated that in the region $|b|>25^{\circ}$, no galactic contribution was detected. This indicates that our CMB upper limits were not contaminated by foreground emission, though any contamination would lead us to overestimate the upper limit rather than underestimate it. 
Keating et al.(2001) presents a summary of the data analysis procedure and the results from the 2000 season. The limits on the large angular scale polarization of the CMB are $T_{E}<10 \mu \mathrm{K}$ and $T_{B}<10 \mu \mathrm{K}$ at $95 \%$ conf idence for the multipole range $2<\ell<20$. Not included in these limits is the $(\sim 10 \%)$ calibration uncertainty. We also calculated the limits on $T_{E}$ with $T_{B} \equiv 0 \mu \mathrm{K}$. Integrating the 1-D likelihood curve for $T_{E}$ with $T_{B} \equiv 0 \mu \mathrm{K}$ we find $T_{E}<8 \mu \mathrm{K}$ at $95 \%$ conf idence.

We have also computed the limits that result from simply subtracting the offset from the 25 subsections of data and increasing the on-diagonal elements of the corresponding covariance matrix by a factor of $N_{\text {pix }} /\left(N_{\text {pix }}-1\right)$, corresponding to the loss of one degree of freedom e.g., [10]. While limits obtained from this procedure do not correctly account for the Stokes parameter correlations induced by the offset removal, they do illustrate POLAR's raw sensitivity, and are useful for comparison with previous experiments. Following the notation of [9], the limits on CMB polarization from this procedure are $T_{E}<8 \mu \mathrm{K}$ and $T_{B}<8 \mu \mathrm{K}$ (both $95 \%$ conf idence). Assuming $T_{B} \equiv 0 \mu \mathrm{K}$, we obtain $T_{E}=6 \mu \mathrm{K}$ at $95 \%$ conf idence.

\section{CONCLUSION}

With POLAR, we have demonstrated that the detection of the large angular polarization of the CMB is diff $i$ cult but technologically feasible. Our limits are encouraging and suggest that future large angular scale, multi-pixel, correlation polarimeters (such as SPORT [11]) may have the capability to detect polarization of the CMB at the $\mu \mathrm{K}$ level. A detection would permit the discrimination between heretofore degenerate theoretical predictions. Polarization of the CMB has a unique signature in both real and Fourier space, as well as distinct spectral characteristics. CMB polarization, in conjunction with anisotropy measurements, is one of the most sensitive probes of the ionization history of the pre-galactic medium. This epoch of cosmic evolution is of great interest, and supplemental information from polarization detection will greatly advance our knowledge of the formation of structure in the early universe.

\section{ACKNOWLEDGMENTS}

This work has been supported by NSF grants AST 93-18727, AST 98-02851, and AST 00-71213, and NASA grant NAG5-9194. BK and CO were supported by NASA GSRP Fellowships. POLAR's HEMT amplif iers were provided by John Carlstrom.

\section{REFERENCES}

1. Pospieszalski, M. W., Lakatosh, W. J., Nguyen, L. D., Lui, M., Liu, T., Le, M., Thompson, M. A., and Delaney, M. J., “““ Cryogenically-Cooled HFET Amplifers and Receivers: State-of-the-Art and Future Trends"', in 1992 IEEE MTT-S Digest, edited by L. Kirby, IEEE, Piscataway, NJ, 1992, p. 1369.

2. Wollack, E. J., Jarosik, N. C., Netterfeld, C. B., Page, L. A., and Wilkinson, D., ApJL, 419, L49-+ (1993).

3. Keating, B., Timbie, P., Polnarev, A., and Steinberger, J., ApJ, 495, 580+ (1998).

4. Wilkinson, D., "A Warning Label for Cosmic Microwave Background Anisotropy Experiments", in Particle Physics and Cosmology, Proceedings of the Ninth Lake Louise Winter Institute, edited by A. Astbury et al., World Scientifc, Singapore, 1995, p. 110.

5. Thompson, A., et al., "Interferometry and Synthesis in Radio Astronomy", Krieger Publishing Co., Malabar, 1998.

6. O’Dell, C. W. e. a., IEEE-MTT (2002).

7. Tegmark, M., Phys. Rev. D., 56, 4514-4529 (1997).

8. Bond, J. R., Jaffe, A. H., and Knox, L., Phys. Rev. D., 57, 2117-2137 (1998).

9. Keating, B. G., O’Dell, C. W., de Oliveira-Costa, A., Klawikowski, S., Stebor, N., Piccirillo, L., Tegmark, M., and Timbie, P. T., ApJL, 560, L1-L4 (2001).

10. Wollack, E. J., Jarosik, N. C., Netterfeld, C. B., Page, L. A., and Wilkinson, D., ApJL, 419, L49-+ (1993).

11. Cortiglioni, S., These Proceedings (2001). 